\title{
Identifying Building Footprints in Historic Map Data using OpenCV and PostGIS
}

Keywords: feature extraction, computer vision, historic maps, building footprints, geographical information science (GIS).

Summary: This paper describes a combined GIS and computer vision approach to the extraction of high quality vector building footprints from a series of scanned historic maps (Ordnance Survey County Series maps). The monochrome input maps predominantly use a speckled texture within a closed polygon to indicate the presence of a building. Image processing, segmentation and a manual data cleanup steps are performed, after which the raster data is vectorised and transformed into georeferenced PostGIS geometries (via contours generated using the python OpenCV module). The identification of buildings, joining of sheets and post-processing is then undertaken using Structured Query Language (SQL) queries passed to a PostGIS enabled PostGreSQL database. To date, it has not been possible to fully automate the process, due to original cartographic decisions like printing text over buildings, damage to the original paper maps and technical limitations. However, the semi-automated process described here has yielded high quality building footprints for two map editions covering the study area.

\section{Introduction}

\section{Background}

The House Age Project (HAP) is a long term project which originated within the Registers of Scotland (RoS) and is currently running as a pilot in collaboration with Historic Environment Scotland (HES). Data for the study area, the historic Scottish County of Edinburghshire (Midlothian) [4] is provided by the National Library of Scotland (NLS) Map Library. The longterm ambition of the project is to assign an age (interval) estimate to each building which currently exists in Scotland (to the extent that it is possible to do so) by combining GIS data extracted from scanned historic maps with present-day GIS datasets.

These project aims encompass a number of challenging research problems, which are being investigated by the project participants using GIS, computer vision and entry-level machine learning approaches. One of the challenges is the extraction of building footprints from scanned historic maps to produce derived GIS vector datasets. The extracted building footprints will be tested as input for further analysis aimed at tracking individual buildings across datasets.

An assumption is made that tracking buildings across datasets will require shape-based comparisons. For this reason every effort has been made to develop a methodology which extract vectorised building shapes as completely and accurately as possible. Due to the large amount of available data, an automated method for extracting the data is highly desirable.

\footnotetext{
${ }^{1}$ GIS Team Leader, Registers of Scotland, Edinburgh [marguerite.leriche@ ros.gov.uk]
} 


\section{Tools}

The analysis is conducted using open source tools. The main python libraries used include OpenCV (computer vision), the python bindings for GDAL (Geospatial Data Abstraction Library), rasterio, scikit-image, numpy, re, pickle, glob and psycopg2 (PostgreSQL database adapter for python). The data is further manipulated in a PostgreSQL database with the PostGIS spatial extension enabled. Data is visualized using QGIS and a manual cleanup step is performed using the GIMP graphics editor.

\section{Data}

The source data are manually georeferenced (either by NLS or in a few instances, by the author), 3-channel, 12000 x 16000 pixel scans of the Ordnance Survey's 25-inch-to-the-mile (1:2500) County Series map sheets [1,2], provided by the NLS in the GeoTIFF (Geographic Tagged Image File) format. Images are reproduced with permission of the National Library of Scotland under a Creative Commons Attribution-Non-Commercial-ShareAlike 4.0 International license.

There are four editions/revisions of the County Series Maps available, mapped between 1855 and 1949, covering urban areas across Scotland. The study area, comprises $\sim 35$ map sheets per edition, covering approximately $137 \mathrm{~km}^{2}$. It includes a range of residential and commercial buildings in different configurations - from densely packed urban tenements to sparsely distributed, rural buildings and a range of suburban build patterns in between.

This paper describes processing of two editions of the $2^{\text {nd }}$ revision, dated 1905-06 and 191213 respectively, wherein buildings are indicated using a speckled texture fill. Each map sheet has been assigned a unique identification number by the NLS. During processing, this reference number is retrieved from the filename and permanently associated with the data extracted from the map sheet. The NLS also provides index shapefiles containing polygons which correspond to the clipped boundaries of each map tile for each edition [3]. The attribute table of the index shapefile contains additional metadata such as survey and publication dates associated with the original maps.

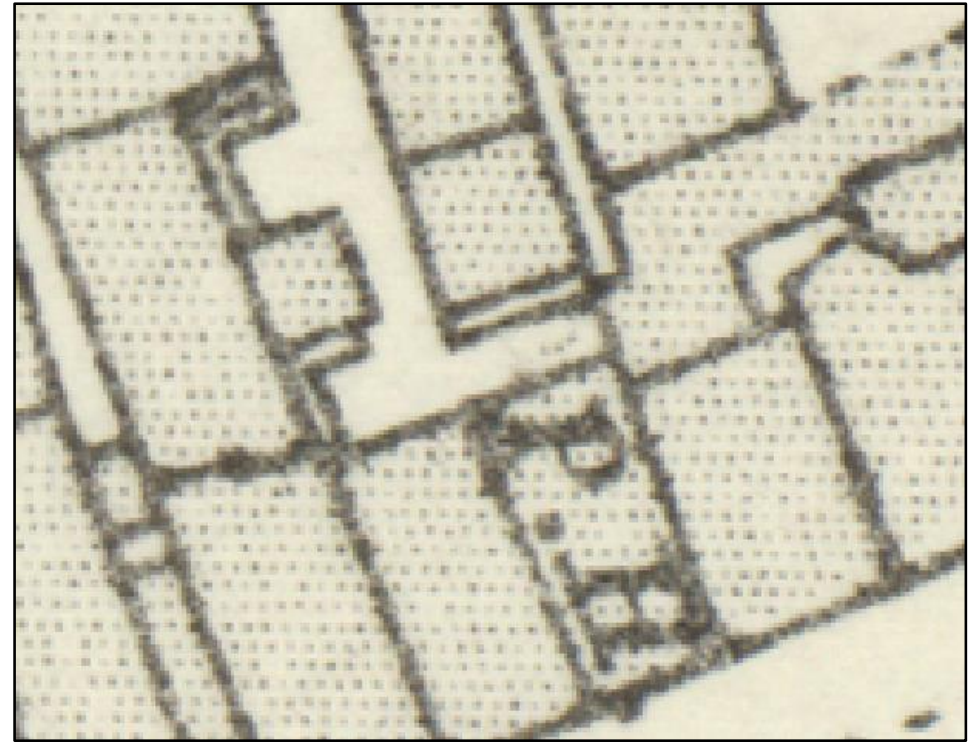

Figure 1. Buildings indicated with a speckled fill (OS County Series) 


\section{Methodology}

\section{Preprocessing}

The GeoTIFF image metadata standard [6] expands the .TIFF image format to allow for georeferencing information to be stored in the image header. This allows spatially enabled software (e.g. QGIS) to display the image correctly using a geographic or projected map coordinate system.

The OpenCV Library uses an image coordinate system and does not copy geo-referencing information from the header when writing a new .TIFF file. Therefore, as a first step, the georeferencing information attached to each map sheet is retrieved using the rasterio transform function, and the resulting python object is saved for later re-use using the python pickle module. As the input data is monochromatic, the initial approach falls into the category that can be described as edge-detection [9]. The input images have a bimodal distribution of pixel values, with the two peaks corresponding to the white background paper and printed black ink. Otsu's binarisation algorithm, which binarises the input image at an automatically calculated threshold (between the two modes) was found to yield the best result for this data.

The threshold value is calculated for each individual sheet, which accommodates variations in the amount of ink laid down on different map sheets as well as variations in scanning. The calculated value is shifted by a small amount before it is applied, so that light gray pixels are sliced to the same binary category as dark gray/black pixels. This is to retain as much information as possible about the original application of ink to paper, and to avoid creating small gaps in lines.

Printed pixels with the same value as the darker noise in the pixels which represent the background paper, are lost during the thresholding step. A number of options for image smoothing/noise removal were tested, but it was found that noise removal degrades the speckle texture.

The thresholded raster image contains a large number of unwanted map features, symbol and labels which are detected as contours by the OpenCV findContours() function. These include trees, boundary lines and a variety of other map symbols. Most problematic are symbols (e.g. archways) and letters (especially hollow letters like 'o', 'p', 'B' etc.) which are printed over, fused to the inside, or bisect the contours which represent buildings. This phenomenon is very well described in the literature [8,9]. As any building identifiers are assigned post-extraction, it is not possible to automatically identify which polygons constitute the separate parts of bisected or fragmented buildings.

Some attempts have been made to remove the unwanted features in a targeted manner (e.g. K Nearest Neighbour algorithms, Haar patterns, approaching others with more computing power for assistance) but their removal remains resistant to automation.

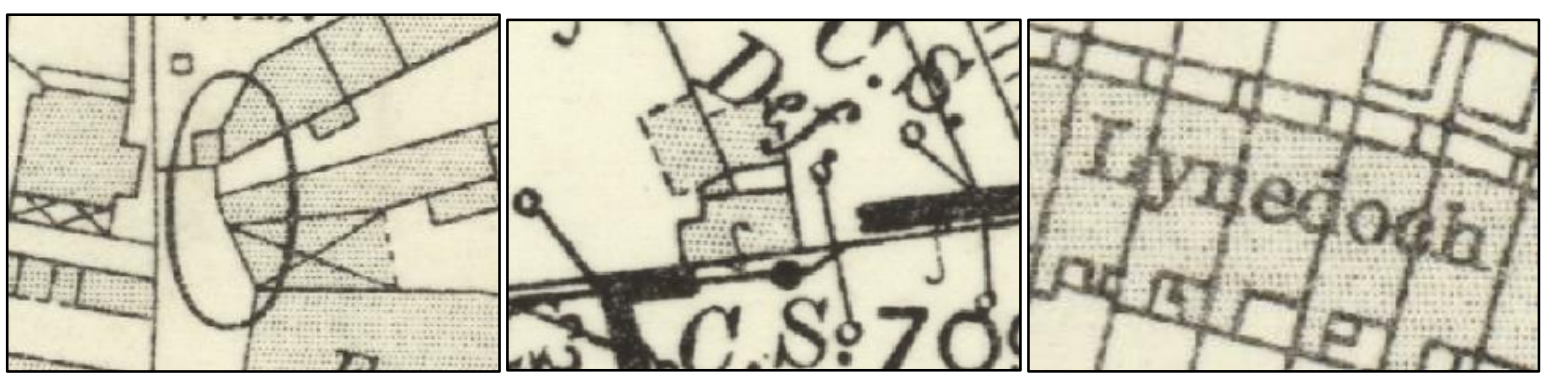

Figure 2. Examples of unwanted overprinted and fused features 
For example, no attempt has been made to automatically reconstruct the 'walls' behind fused symbols after they have been removed. An acceptable compromise between speed and accuracy is to inspect contours for size and roughness (perimeter/area) and to mark those which are unlikely to be speckles or buildings for removal. Fused features are resolved in the manual step.

Due to paper warping, the map neat line is clipped in some places, leaving 'opened' buildings in the georeferenced raster images. To prevent buildings from being lost at the sheet edges, a new neat line is constructed by obtaining the rotated bounding box of all the data within the map sheet.

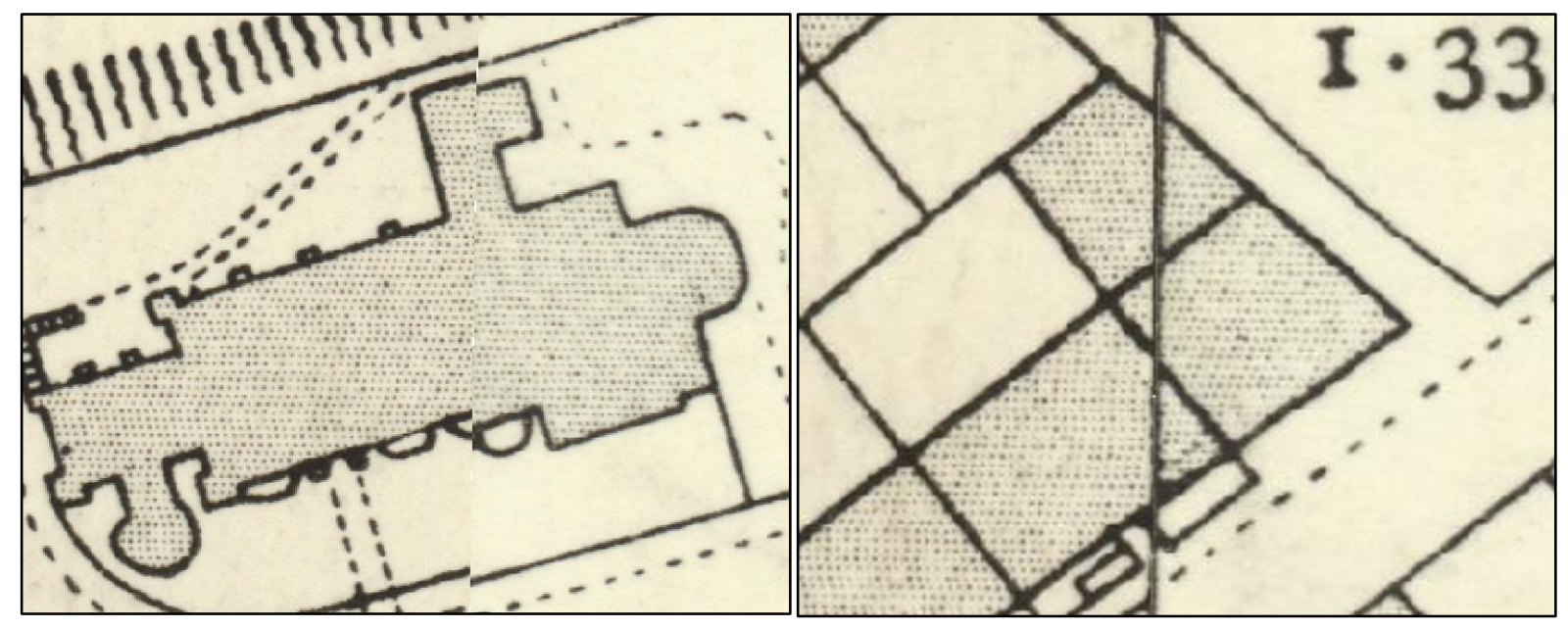

Figure 3. Lost (left) and retained (right) neatlines on sheet edges

The data contains a number of temporary glasshouses and canopied structures whose walls are indicated with dashed lines. Glasshouses are not extracted at this time, but buildings indicated with dashed lines are reconstructed and extracted. The first step in reconstructing dashed walls is to isolate dash-like contours in close proximity to speckles. The centroid of each of these contours is then connected to the nearest centroid within a threshold distance with a line. The shape of dashes in the dataset is not always well defined enough to reliably capture their orientation, making it difficult to draw closed shapes or extend the reconstructed lines to join up with neighbouring structures. Any unclosed shapes and unwanted artifacts created by the dashed line reconstruction are resolved in the manual step.

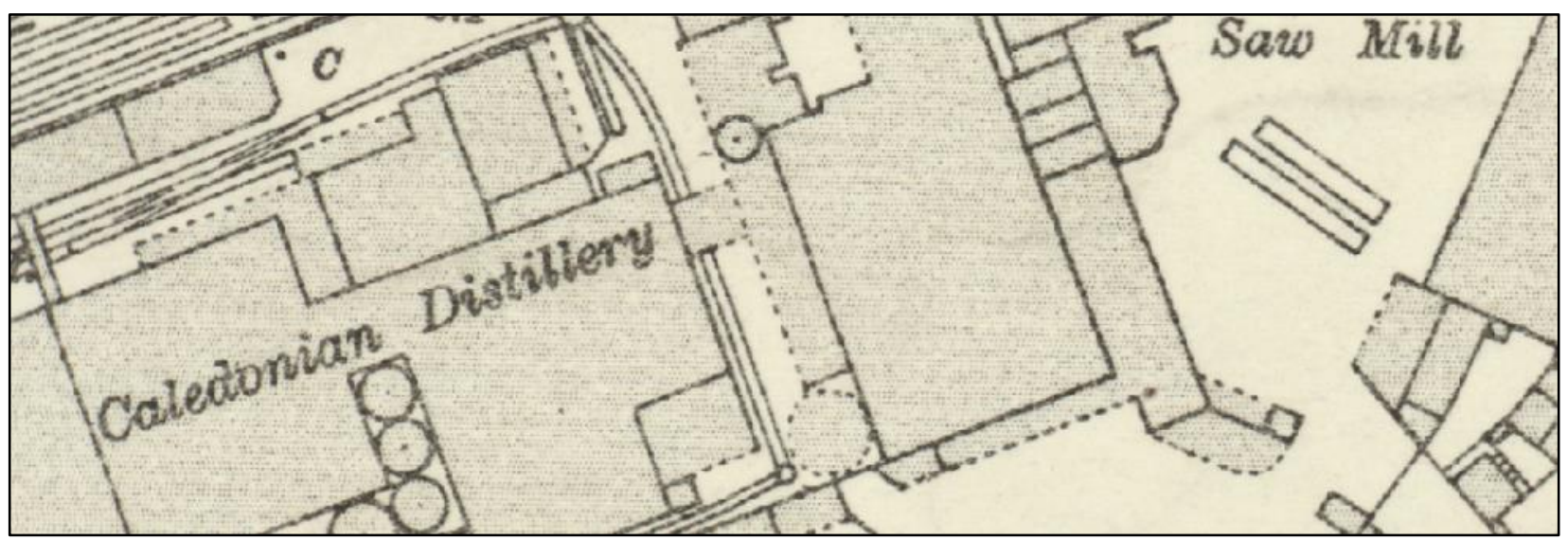

Figure 4. Structures indicated with dashed lines 
The outputs from the steps above are then used to create a segmented image where speckles, potential buildings, potentially unwanted features and partially reconstructed dashed walls are differentiated by colour.

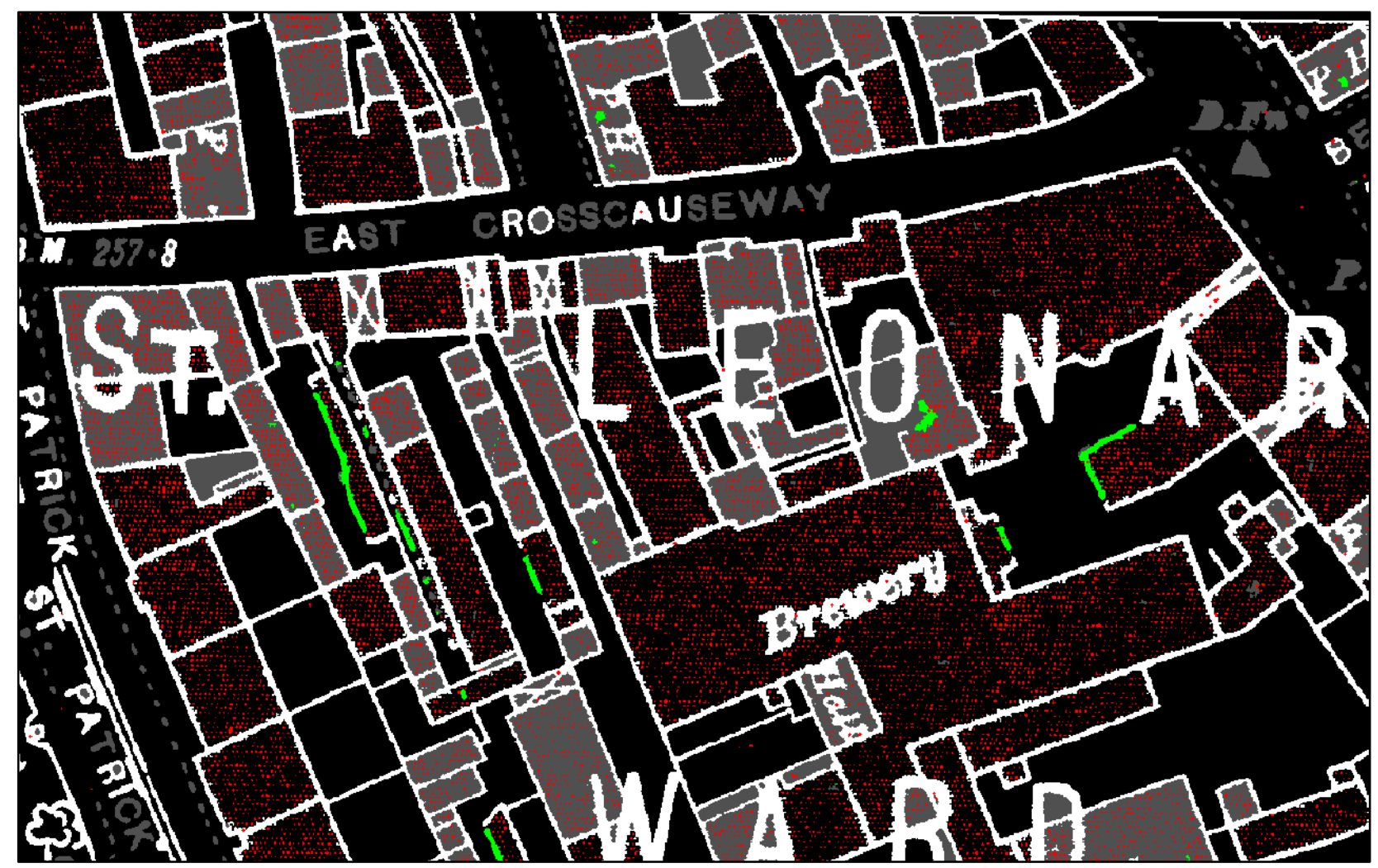

Figure 5. Segmented image with speckles (red), areas for removal (gray), probably walls (white) and reconstructed dashes (green)

\section{Manual Step}

Each coloured, segmented map sheet is inspected and edited manually using the GIMP software editor. This means that the input data is altered from this point onwards. However, the alterations can be made in different colours (e.g. yellow for changes which need to be retained and dark blue for areas which need to be discarded) to make the manual step transparent and auditable.

Features that have been classified as unwanted (dark gray) are checked. Any misclassified features are filled in a light colour, using the bucket tool. This issue may affect very small freestanding buildings or freestanding buildings with gaps in the outline.

Large gaps in lines are also closed. The OpenCV distance transform and watershed algorithms are highly effective at closing small gaps. However, some of the narrowest buildings are narrower than some of the largest gaps in this dataset. It is not possible for the watershed algorithm to distinguish between these cases, which results in spurious lines being drawn across narrow buildings. The algorithm also causes a noticeable rounding of corners beyond that which is present in the input data. In this instance, as we wanted to capture the shape of buildings as faithfully as possible, it was decided to close gaps in the manual step.

Hollow fused text, and features which completely bisect buildings are scored through to break up the closed shapes. Some non-hollow text and the scored-through remnants can be left 
as these and other artifacts it will be reduced to GIS geometry errors once the data has been sent to the PostGIS database, where it can be automatically repaired. However, they will in some cases leave a trace in the form of a small dimple in the final building outline.

Some buildings are so small that their speckles are all fused to the lines and the clone stamp can be used to resolve this.

The bright green reconstructed dashed lines are checked for open segments, which are closed with the crayon tool. Some areas with heavily printed, joined-up speckles may contain artifacts created by the dash reconstruction and these are removed.

There are rare locations where so many features are overprinted that it is not possible to make a (subjective) decision about where the building outline is. These are left as-is, for extraction to fail.

The time taken per sheet varies from 10 minutes for a more rural sheet to 2 hours for a dense city center sheet. This requires a reasonable investment of time and sustained concentration and may not be feasible for a large number of maps of dense urban areas. However, it only needs to be done once per dataset and in this instance did not take as long as developing a training dataset for testing machine learning approaches.

\section{Extraction and Vectorisation}

The manually corrected image is segmented again using colour thresholds. This yields two new binary arrays, one consisting of the speckles and the other of the larger line features which need to be retained. Contours which are not potential buildings or speckles are discarded.

The OpenCV contours for the speckle array is inspected to capture the first node of each contour. The contour node is transformed using the pickled affine transformation and wrapped in a text string which resembles the Well-Known-text (WKT) spatial data format. The WKT string is then passed to the PostGIS database using the psycopg2 python module, within a command to generate PostGIS geometry from text (ST_PointFromText()). There can be many speckles in a map tile (hundreds of thousands) and as the work is being conducted on a standard developer laptop, disk speed was a factor in the processing time.

The line features array undergoes further processing. The GDAL sieve algorithm is used to remove pixel 'burrs' with a single 8-connection to the lines. These burrs are caused by speckles that have been fused/partially printed over the lines. They may cause the final building edges to be slightly offset from the center of the line in the input image.

The array containing the lines is skeletonized using a straightforward application of the scikit-image skeletonisation algorithm. This yields a skeleton with a large number of unwanted spurs which are difficult to remove using a raster/array approach. However, once the contours are captured and sent to the PostGIS database, these spurs are reduced to GIS geometry defects which can be easily repaired using standard functions like ST_MakeValid().

The skeletonized contours are retrieved and a basic validity check is performed, to confirm that it has at least three nodes. They are then transformed, formatted as WKT and posted to the PostGIS database. From this points onwards the analysis is conducted using SQL and a GIS approach. 


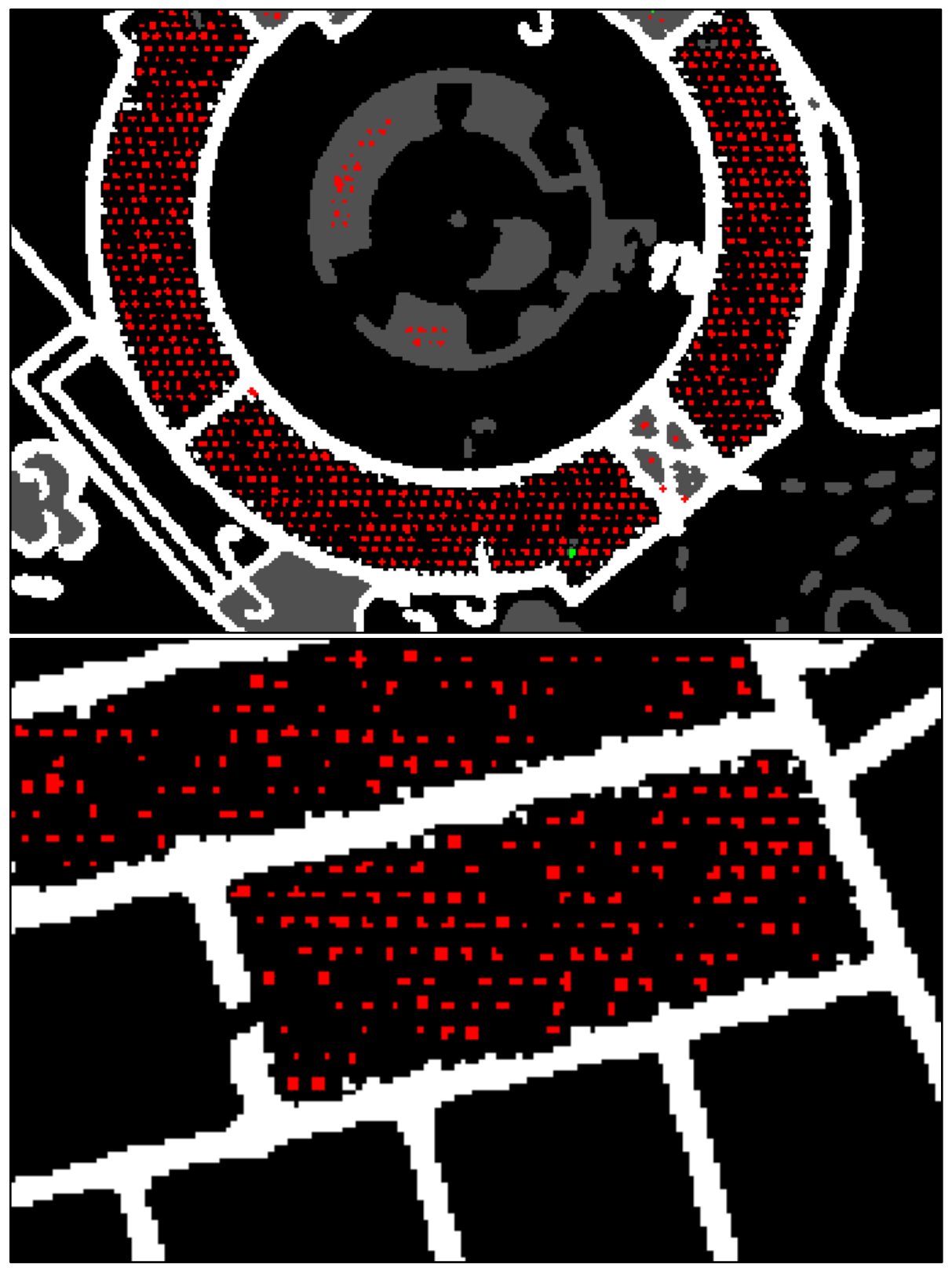

Figure 6. Manual corrections, building incorrectly classified as unwanted (gray), overprinted archway (white ' $X$ '), artifact from dash-reconstruction (green) and gap in line (right image)

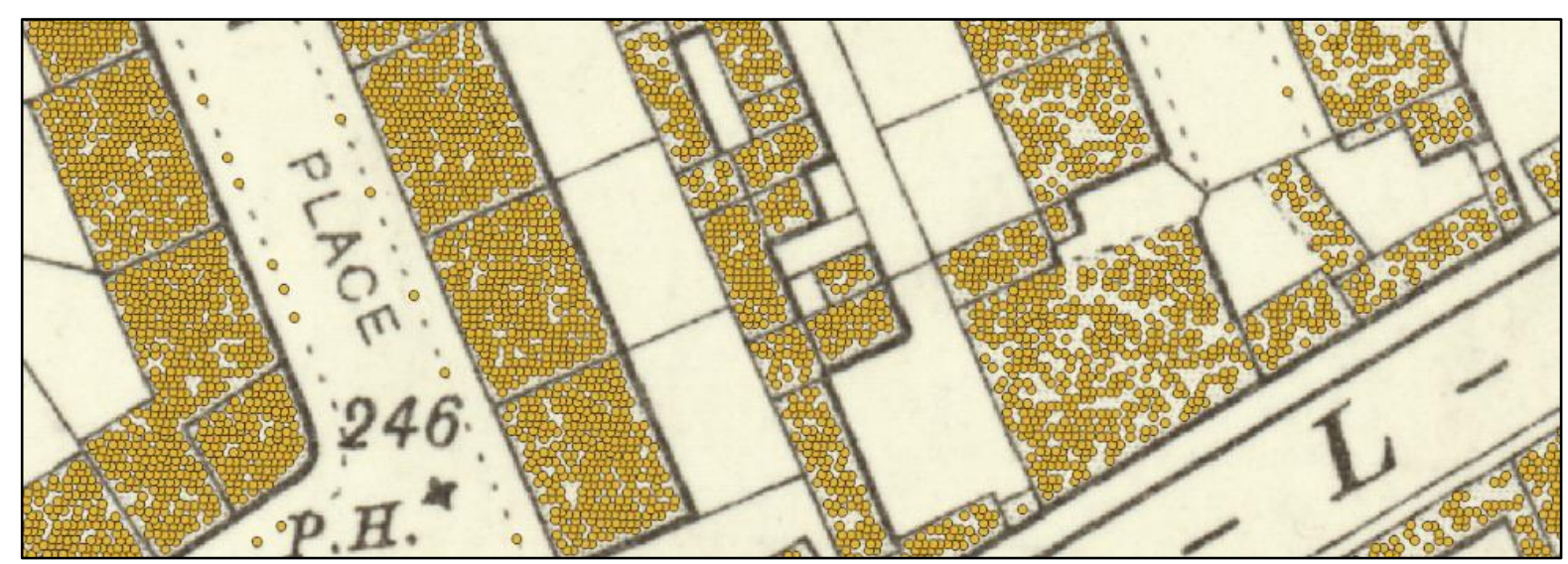

Figure 7. Speckles as vector point geometries after vectorisation 

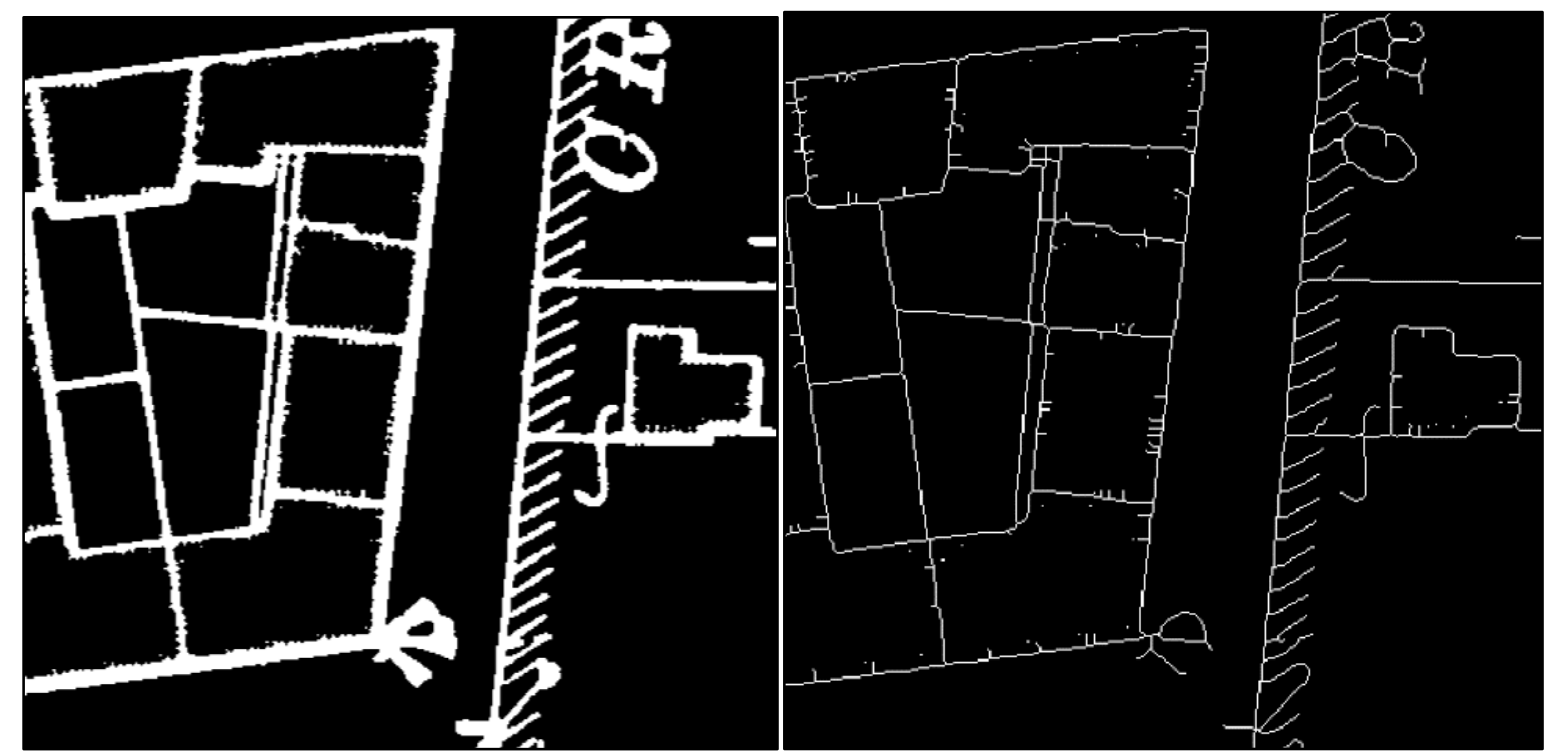

Figure 8. Lines before and after skeletonisation

\section{Building Identification}

At this point the PostGIS geometries contain invalid geometries, as defined by the Simple Feature Standard [7], which is not implemented in OpenCV contours. It is important to note that these geometry defects may, in some instances, represent useful information which will be lost once the geometry is repaired.

The newly created PostGIS geometries are reduced to their smallest constituents using ST_Dump() and then repaired using ST_Makevalid(). A tiny buffer followed by a tiny negative buffer resolves any remaining spurious geometries caused by skeletonisation and small unwanted features that were not removed in the manual step. New fields are added, the area of each polygon is calculated and those that are considered too large or too small to be buildings are discarded. A standard GIS point-in-polygon operation is performed to count the number of vectorised speckles inside each polygon.

The ratio of point to area is used to determine whether a given polygon is likely to be a building or not. An arbitrary threshold was chosen based on examination of the data and applied to all the map sheets, but equally it could be possible to calculate an optimal threshold for each sheet.

Once all the map sheets have been processed and copied to a master table, a primary key is added. At this stage the geometries are all simple, single part polygons consisting of a single ring. The table is inspected using QGIS. Some extremely large or oddly shaped buildings, e.g. buildings with a very large (speckle-free) central courtyard, may have been dropped. These can be retrieved from a back-up of the unfiltered set. 


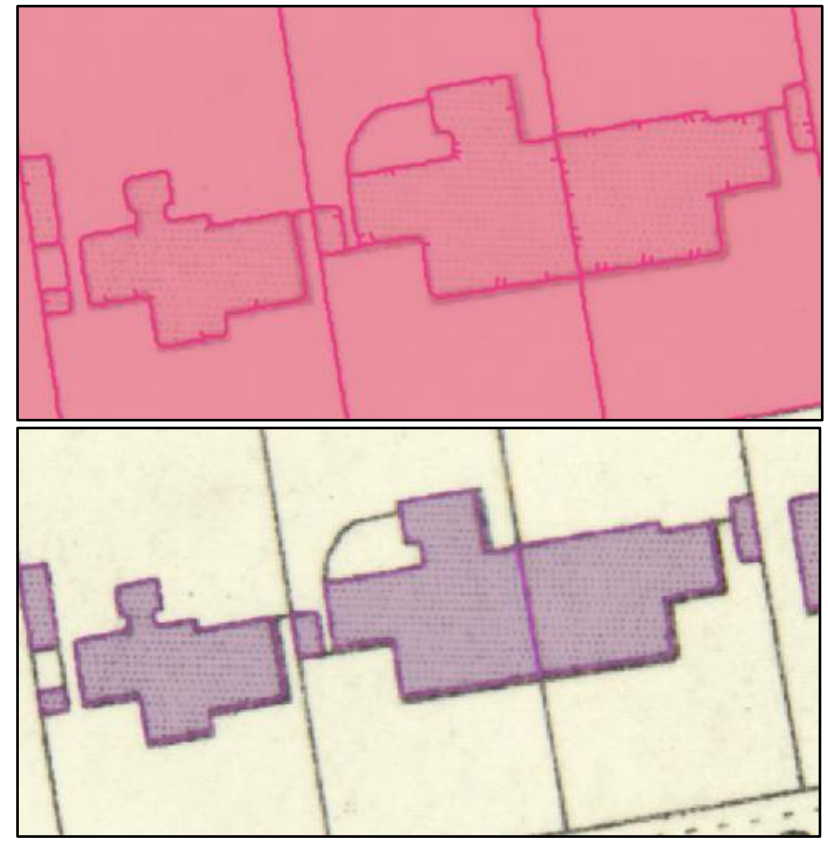

Figure 9. PostGIS vector geometry before and after repair and filtering

\section{Post-processing}

OpenCV creates two contours for each feature - one child inside the white edge and one parent outside. For freestanding buildings, this results in two overlapping polygons with very similar area and ratio properties. It is possible to remove one of them using SQL queries, and a choice was made to retain the inner one. However, making an automated choice between two slightly different shapes will have implications for any future shape-based analysis. Some cases may need to be resolved manually.

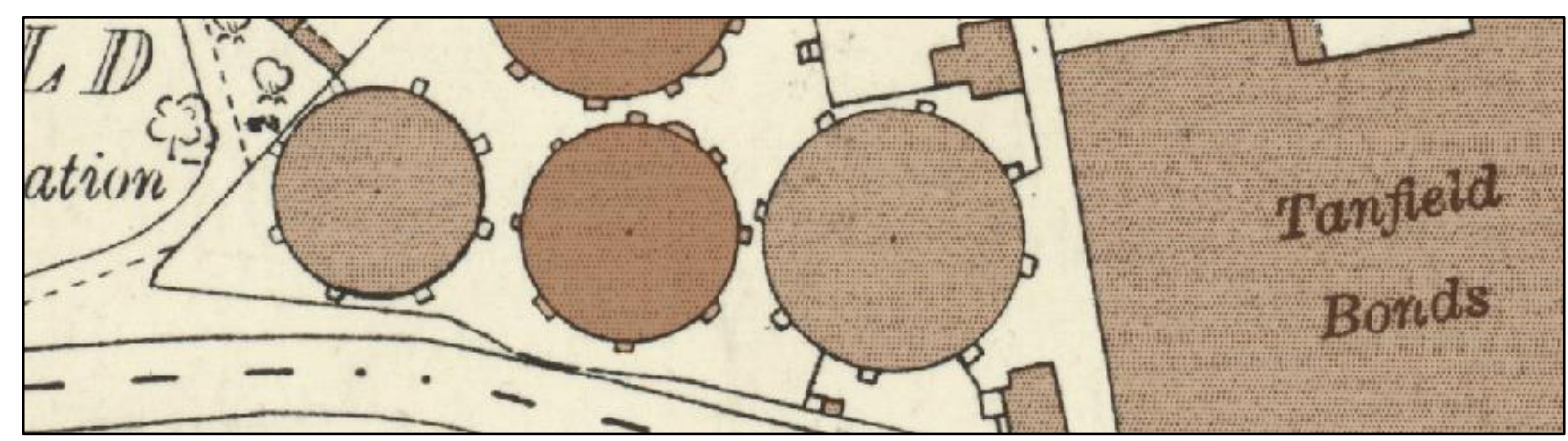

Figure 10. Overlapping polygons (darker) caused by parent/child contours

Sheet edges are joined by identifying all building polygons within a search distance of the neat line/index polygon edge. The geometry of buildings with different tile numbers are then snapped to each other and their union calculated (with ST_Union()). A slightly more complex query is required to calculate the union for buildings which fall on the meet point of four corners. The results are varied and the resulting shapes may not be suitable for further shape analysis. In some locations, the union is excellent, but ST_Snap tends to yield unexpected results where the gap between the sheets are large or the buildings are not perfectly aligned. Where the gap is very large due to severe paper warping or imperfect geo-referencing, they may fail to join. In the dataset 
tested there were 37 good unions (although they did not necessarily yield a good shape), 58 correct unions with defective geometries, 6 unions were missed and 28 false unions were made. Further work will be required.
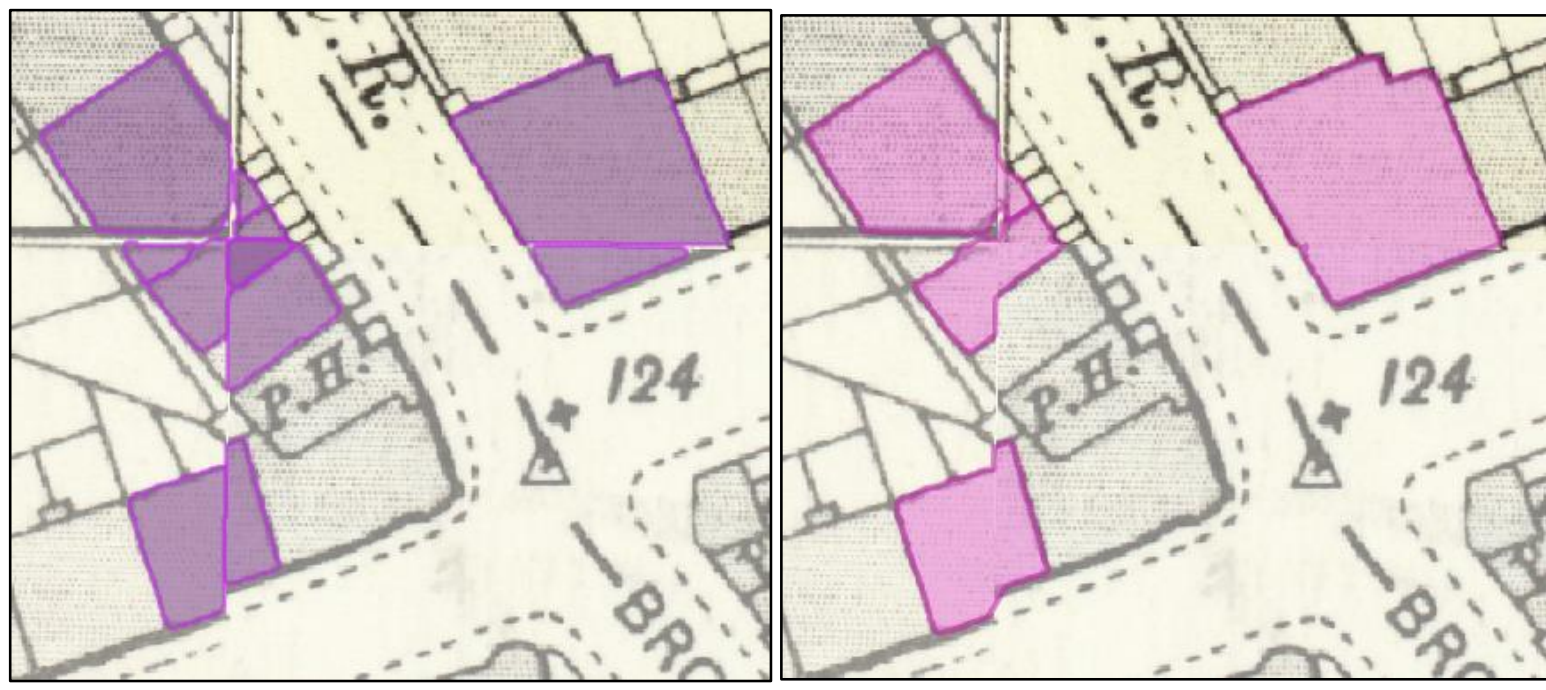

Figure 11. Joining sheet edges

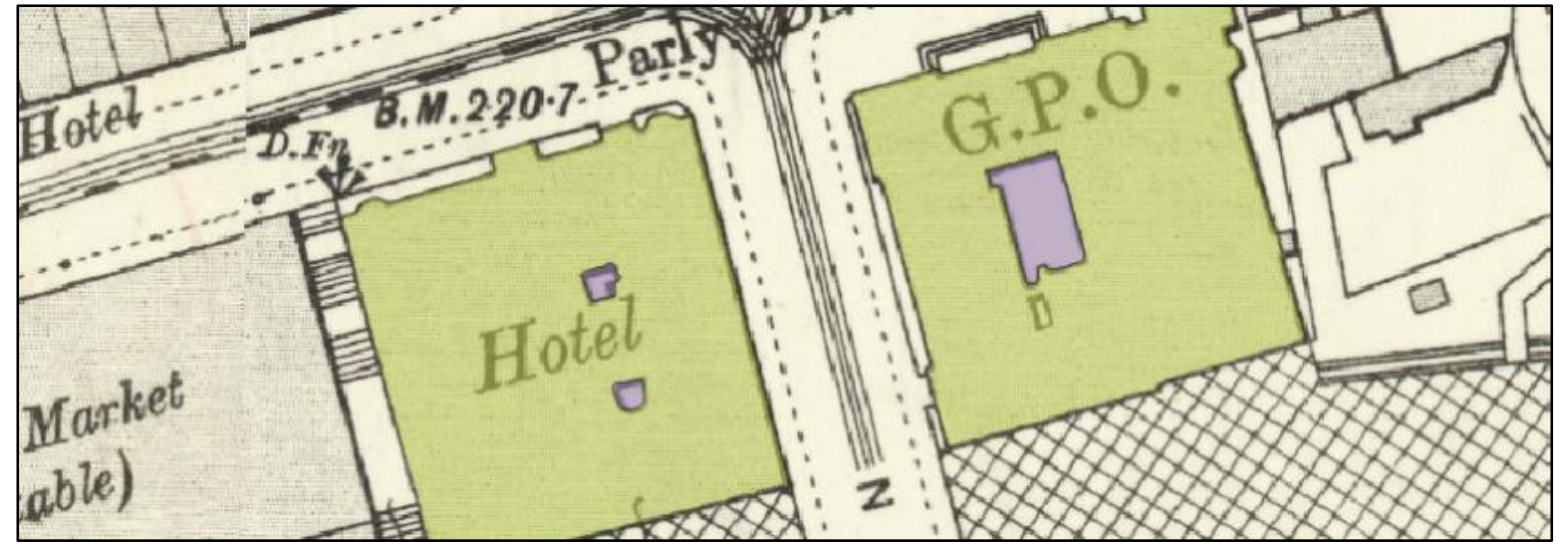

Figure 12. Adding interior rings for courtyards

The final step is to detect small, low ratio courtyard polygons using a polygon-in-polygon test. They are then cut out of building polygons as an inner ring. If hollow text is not removed before vectorization, they may cause spurious inner rings to be created in this step. Metadata is copied from the index layer to each building polygon extracted from the sheet.

\section{Results}

A confusion matrix was created using a sample of four ( $\left(1^{\text {st }}\right.$ edition of the second revision) map tiles covering Edinburgh City Centre and Arthur's Seat. The results were inspected at a viewing scale of 1:750. 73087 OpenCV contours were sent to PostGIS as potential buildings. 18321 polygons were eventually classified as buildings and 54766 discarded as not buildings. There were 369 false negatives and 435 false positives. The majority of the false negatives are very small buildings which fell below the selected size threshold. The majority of false positives are similarly small polygons just over the size threshold, which form part of complex symbology like beach shingle or embankments where some 'speckles' may be present. 
Table 1. Confusion matrix

\begin{tabular}{|c|c|c|c|c|}
\hline $\begin{array}{l}\text { Actual class (across) / } \\
\text { Predicated class (down) }\end{array}$ & Building & $\begin{array}{l}\text { Non- } \\
\text { building }\end{array}$ & \multirow{4}{*}{$\begin{array}{l}435 \text { False positives: } \\
32 \text { sheet edge artifacts } \\
386 \text { tiny artifacts } \\
17 \text { yard/courtyard polygons } \\
\text { merged with building } \\
\text { polygons }\end{array}$} & \multirow{4}{*}{$\begin{array}{l}369 \text { False negatives: } \\
21 \text { dashed buildings lost } \\
4 \text { buildings fragmented } \\
246 \text { tiny buildings lost } \\
59 \text { buildings lost to gaps } \\
2 \text { slivers of buildings lost at } \\
\text { sheet edges. } \\
37 \text { buildings lost as they } \\
\text { were incorrectly } \\
\text { misclassified as unwanted } \\
\text { in preprocessing }\end{array}$} \\
\hline Building & 17886 & 435 & & \\
\hline Non-building & 369 & 54397 & & \\
\hline \multicolumn{3}{|c|}{$\begin{array}{l}\text { Accuracy }=(17886+54397) / 73087=98 \\
\text { Misclassification }=(435+369) / 73087= \\
\text { False positive rate }=435 / 18321=2.4 \% \\
\text { False negative rate }=369 / 54397=0.7 \%\end{array}$} & & \\
\hline
\end{tabular}

It is possible to extract the majority of buildings without a manual step, but buildings are lost in particular where overprinted features and gaps in the lines are present. An earlier version of the methodology (without the manual step, but including a watershed algorithm) identified 19066 features as buildings in the four sample map sheets, of which 1916 were false negatives. 425 dashed buildings were lost and 504 buildings were fragmented into two or more polygons.

Buildings affected by overprinted text are not randomly distributed - they tend to occur in more densely built up areas and a disproportionate number of public houses (pubs) are affected, as they tend to be small, oddly-shaped street corner buildings and have the text 'P.H.' overprinted in relatively large capital letters [5].

The manual step brings the overall extraction rate to a satisfactory $98.9 \%$. However, the extraction rate is not the only factor to consider when judging the quality of the polygons and their suitability as input for shape analysis.

The boundaries of the extracted polygons are well-contained within the lines of the original scanned map image and generally falls visually on the centerline. The boundaries of adjacent buildings are coincident. Corners are slightly rounded and the geometries are highly complex, with a very large number of vertices, as the extracted lines follow the jagged pixel edges of the input raster data. Due to the complexity of the geometry, the output file size is large and subsequent processing slower than desired. Further work on simplification/generalization is being undertaken and it is not clear yet whether the outputs are suitable for further shape-based analysis.
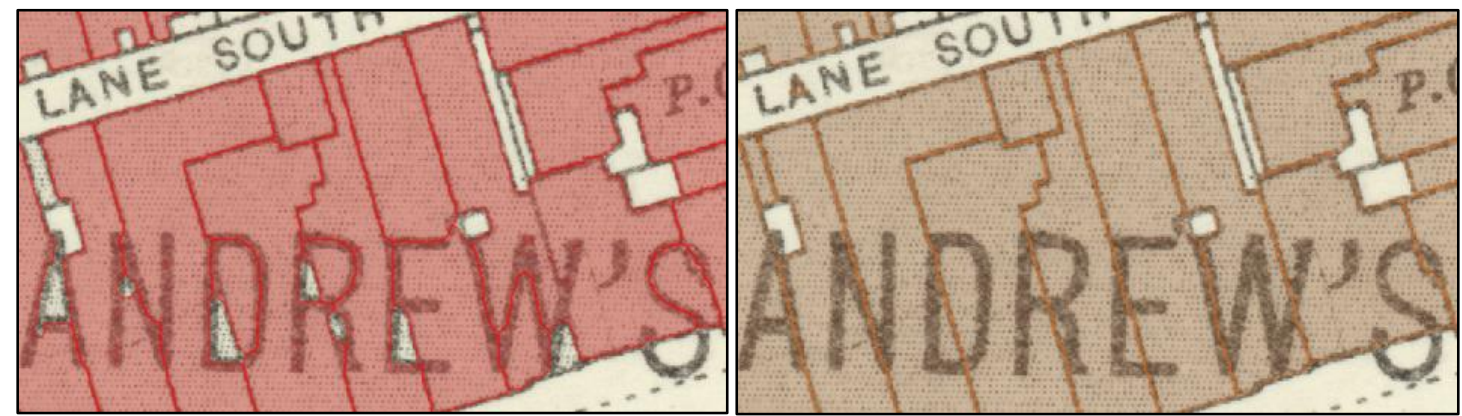

Figure 13: Effect of overprinted text without (left) and with (right) the manual step 


\section{Discussion}

A number of practical factors are reflected in the methodological choices described above. The focus of the work is to produce datasets, rather than to solve all issues around automation. These unresolved issues include effects caused by some of the original cartographic choices, e.g. text printed across buildings and buildings indicated by dashed lines; as well as issues caused by the age of the original paper map sheets, e.g. damage and warping at sheet edges. For this reason, a semi-automated solution is being considered as part of the House Age Project. A manual step resolves the issues that are resistant to automation with a high degree of success. However, it does involve making (auditable) changes to the input data, which introduces a subjective element into the analysis.

The hardware available to the project has been limited to high-specification business laptops. The resulting lack of processing power has closed off a lot of potentially fruitful avenues of inquiry, from simple options like searching images with a sliding window to more advanced machine learning algorithms. However, it has been possible to implement a combined computer vision and GIS approach which solves the problem of building identification through simple spatial queries rather than processing intensive and complex feature detection methods.

The Well-Known-Text data format offers an easy and flexible way of transforming OpenCV contours to GIS geometries. It has an advantage over the shapefile format in that it allows OpenCV contours to be imported into a GIS system as-is, which may include mixed geometry types and invalid geometries (i.e. which do not confirm to the Simple Features Standard). Geometry defects are automatically removed during shapefile creation, so using WKT as an intermediary format allows for much greater control over the data during the transformation of OpenCV contours into valid GIS geometries.

PostgreSQL/PostGIS database tables and Structured Query Language (SQL) offers flexible methods for establishing spatial relationships between selected geometries in the same or adjoining map sheets. The geometries created using this approach are highly complex (they contain a lot of nodes) and this is already causing unexpected results to occur in steps relating to joining parts of buildings across sheet edges. Work on generalization is being undertaken.

\section{Conclusion}

To date, it has not been possible to develop a fully automated process for extracting building footprints from the OS County Series Maps used in this pilot study. However, a semi-automated process which makes use of a spatial approach to building identification has yielded high quality building footprints, with very little processing power and relatively easy-to-understand tools.

\section{Acknowledgements}

I would like to thank James Crone (EDINA), the Scottish Government's Data Science Accelerator Programme, Mike Middleton (Historic Environment Scotland) and Chris Fleet (National Library of Scotland) for their support.

\section{References}

National Library of Scotland (2020). Ordnance Survey Maps - 25 inch $1^{\text {st }}$ edition, Scotland, 1855-1882. In digital form https://maps.nls.uk/os/25inch/index.html 
National Library of Scotland (2020). Ordnance Survey Maps -25 inch $2^{\text {nd }}$ and later editions, Scotland, 1892 - 1949. In digital form https://maps.nls.uk/os/25inch-2nd-and-later/index.html

National Library of Scotland (2020). Ordnance Survey map - Survey and revision dates for county series mapping, 1843-1943. In digital form

https://maps.nls.uk/os/county-series/dates.html

National Library of Scotland (2020). Counties of Scotland, 1580s-1940s.

In digital form https://maps.nls.uk/counties/\#edinburghshire

National Library of Scotland (2020). Ordnance Survey Abbreviations.

In digital form https://maps.nls.uk/os/abbrev/p.html

Open Geospatial Consortium (2020). OGC GeoTIFF Standard.

In digital form https://www.opengeospatial.org/standards/geotiff

Open Geospatial Consortium (2020). Simple feature Access - Part 1: Common Architecture. In digital form: https://www.opengeospatial.org/standards/sfa

K. Tombre, S. Tabbone, L. Pelissier, B. Lamiroy, and P. Dosch. (2002). Text/graphics separation revisited. Proceedings of the 5th International Workshop on Document Analysis Systems V (DAS'02), D. P. Lopresti, J. Hu, and R. S. Kashi, Eds., Springer-Verlag, London, UK, 200-211. https://doi.org/10.1007/3-540-45869-7_24

Yao-Yi Chiang, Stefan Leyk, and Craig A. Knoblock. (2014). A survey of digital map processing techniques. ACM Computing Survey 47, 1, Article 1. https://doi.org/10.1145/2557423

Yao-Yi Chiang and Craig A. Knoblock. (2013). A general approach for extracting road vector data from raster maps. International Journal on Document Analysis and Recognition (IJDAR) 16, 55-81. https://doi.org/10.1007/s10032-011-0177-1 